\title{
THE LATE SEQUELAE OF PNEUMOCOCCAL MENINGITIS
}

\author{
BY
}

\author{
HONOR V. SMITH, R. M. NORMAN, and H. URICH
}

From the Radcliffe Infirmary, Oxford, and the Burden Neuropathological Laboratory, Frenchay Hospital, Bristol

Before the advent of effective chemotherapy, pneumococcal meningitis was not only a fatal disease, but one that ran its full course with great rapidity. Cases invariably came to necropsy within 10 days of the onset of the illness and usually very much sooner (Cairns and Russell, 1946). Although sulphonamides alone had little effect on the ultimate mortality the infection was often partially suppressed and the speed of the disease slowed down. When, however, penicillin became available the prognosis was revolutionized. In 1943 a trial of penicillin in the treatment of pneumococcal meningitis was initiated at Oxford by the late Sir Hugh Cairns: the value of penicillin was quickly established (Cairns, Duthie, Lewin, and Smith, 1944) and by combining it with sulphonamides the mortality was reduced to the order of $10 \%$ (Smith, 1951). Moreover, in certain unsuccessful cases death only occurred after illness lasting weeks or months.

In parallel with this modification of the clinical picture the necropsy findings also altered, and particularly those with regard to the vascular changes (Cairns and Russell, 1946). In 1944, at a time when the limits to what might be achieved with chemotherapy were still unknown, a child was admitted to the Radcliffe Infirmary, Oxford, who had already been ill with pneumococcal meningitis for some weeks and had developed the syndrome of posterior basic meningitis of infants (Gee and Barlow, 1878). After a long and difficult illness the infection was eradicated and the obstructive element in the hydrocephalus relieved. This was one of the few cases in which useful recovery was not achieved (Smith, Duthie, and Cairns, 1946), and the child survived as an idiot until 1955. We consider that this case merits a full description since, in our experience, it is unique both in regard to the survival period and the necropsy findings, which latter exemplify the end-result of a disease process that has hitherto been studied only in the active stage.

\section{Case Report}

Clinical History (R.I. 27963). - C. S., a girl, was born on November 15, 1942, and died on November 2, 1955.
Pregnancy, delivery, and early development were normal; the child learnt to talk when about 1 year old and to walk two months later. She had no illnesses until May 1, 1944, when she became listless and drowsy. The following day she developed signs of meningeal irritation and was admitted to hospital where lumbar puncture yielded a purulent fluid containing pneumococci.

The child was treated with sulphathiazole, $\frac{1}{2} \mathrm{~g}$. every four hours. Her condition improved; after a week the dose of sulphathiazole was reduced to $\frac{1}{2} \mathrm{~g}$. every six hours and stopped altogether two days later. At that time the cerebrospinal fluid still contained 800 leucocytes per c.mm. of which $95 \%$ were polymorphonuclears. Three days later the child was still feverish and pneumococci were isolated on blood culture. For the next week she received $\frac{1}{2} \mathrm{~g}$. of sulphathiazole four hourly and for three days this was supplemented by intramuscular injections of penicillin to a total dose of 154,000 units. Two subsequent blood cultures were sterile, but in spite of this the child grew steadily worse, the fever persisted, the head retraction increased, and swallowing became difficult. On June 4 she was transferred to the Radcliffe Infirmary, Oxford, under the care of Sir Hugh Cairns. On the morning of her journey she had several fits which were predominantly right sided.

On admission the child was in a deep coma, very pale and dehydrated, and her respirations were rapid and irregular. The pupils were fixed and she presented a picture of decerebrate rigidity with arched back and spastic limbs; while the right limbs were held firmly in extension, the left alternated slowly between flexion and extension. The optic fundi were normal, the fontanelle closed, and the skull measured $45 \mathrm{~cm}$. in circumference.

She was given a blood transfusion and 50,000 units of penicillin by intramuscular injection. Bilateral frontal burr holes were fashioned and both lateral ventricles tapped. They were greatly dilated and found at a depth of $3.5 \mathrm{~cm}$., while the intraventricular pressure was raised to at least $280 \mathrm{~mm}$. of water. The ventricular fluid contained numerous pus cells and Gram-positive diplococci which failed to grow on culture. When the procedure was repeated the following day, pneumococci type 19 were isolated on culture of the C.S.F.

Seven thousand units of penicillin were instilled into the ventricles and for the next week the intracranial pressure was controlled by twice daily ventricular and cisternal taps during which penicillin was instilled in 


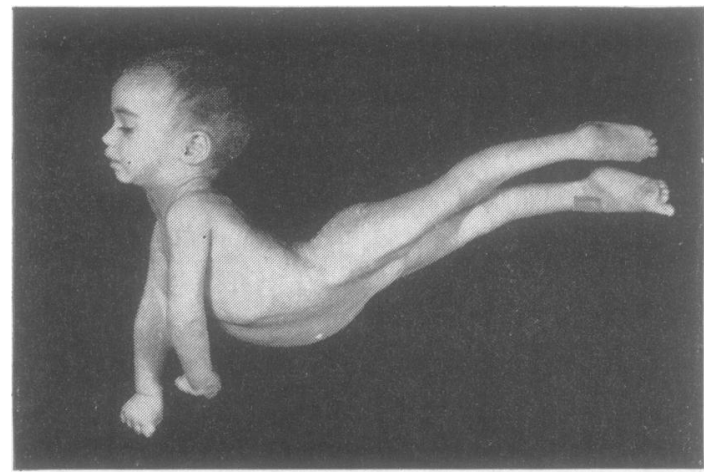

Fig. 1.-Clinical photograph of the patient after suboccipital craniotomy: decerebrate posture with extensor rigidity and opisthotonus.

doses of approximately 5,000 units. This was supplemented by intramuscular penicillin and oral sulphadiazine. On this régime the pneumococci disappeared permanently from the C.S.F.

The intracranial pressure remained high and after one week a posterior fossa decompression was performed and a drain inserted into the cisterna magna. So long as the drain remained in position the intracranial pressure was low, but as soon as it was removed the pressure rose again to dangerous heights. Ventriculography was carried out and revealed a severe symmetrical dilatation of the ventricular system and, in addition, a large cyst or diverticulum occupying most of the right frontal lobe. Only a few bubbles of air passed into the cisterna magna; this was interpreted as evidence of an obstruction to the egress of C.S.F. from the fourth ventricle.

The posterior fossa was explored and inflammatory adhesions were found between the cerebellar tonsils and over the posterior medullary velum leaving only a pinpoint aperture in the roof of the fourth ventricle which was enlarged to about $7 \mathrm{~mm}$. in diameter. The presence of a partial block was thus confirmed, but it was thought that there might well be a further obstruction at the level of the cisterna ambiens.

After operation, there was little change in the child's condition. The decerebrate posture was now extreme and interference of any kind, even noise, would provoke violent spasms of extensor rigidity in which the respirations would become extremely rapid and stertorous (Fig. 1).

At the beginning of August the child began to improve. Crying and swallowing returned and the extensor spasms became less frequent. She was discharged from hospital on November 4. At that time her nutrition was good and the C.S.F. normal in all respects. She could swallow well and would respond to any stimulus by a long drawnout cry. The fundi oculi were normal, the pupils reacted to light, and the corneal reflexes were present. Occasional involuntary conjugate movements of the eye were seen. All four limbs were extended and paralysed. No tendon jerks could be obtained and the plantars were frankly extensor. The decompression was slack and the operation wounds healed.
On subsequent examination in 1946 she was found to have bilateral optic atrophy and a searching nystagmus. Fixed contractions of the joints had developed, but otherwise her condition remained stationary until her death from bronchopneumonia at the age of 13 years.

Necropsy Findings.-The body was that of a small thin girl with severe dorsal scoliosis, fixed flexion contractures of the upper limbs, and extension contractures of the lower limbs.

There is an extensive bronchopneumonia involving the right upper and part of the right lower lobe. The diaphragm shows a large hiatus hernia.

The skull shows bilateral frontal burr holes and a suboccipital craniotomy defect. The dura is normal except for a few firm fibrous adhesions in the posterior fossa.

The fixed brain weighs $377 \mathrm{~g}$., of which the cerebellum and brain-stem account for $81 \mathrm{~g}$.

The frontal lobes, the insulae, and the greater part of both parietal lobes are replaced by a thin, semitranslucent membrane (Fig. 2). Of the parietal lobes, the precuneus and the posterior rim of the paracentral lobule are preserved on the medial surface. On the convexity the superior parietal lobules and the upper and posterior part of the inferior parietal lobules are present. On the left side a shrivelled angular gyrus can be seen; on the right this structure cannot be identified. The convolutional pattern of both occipital lobes appears normal on the convexity as well as on the medial and inferior surfaces. In the temporal lobes the superior temporal gyrus on the left, the superior and middle on the right are thin and shrivelled, but the crowns of the

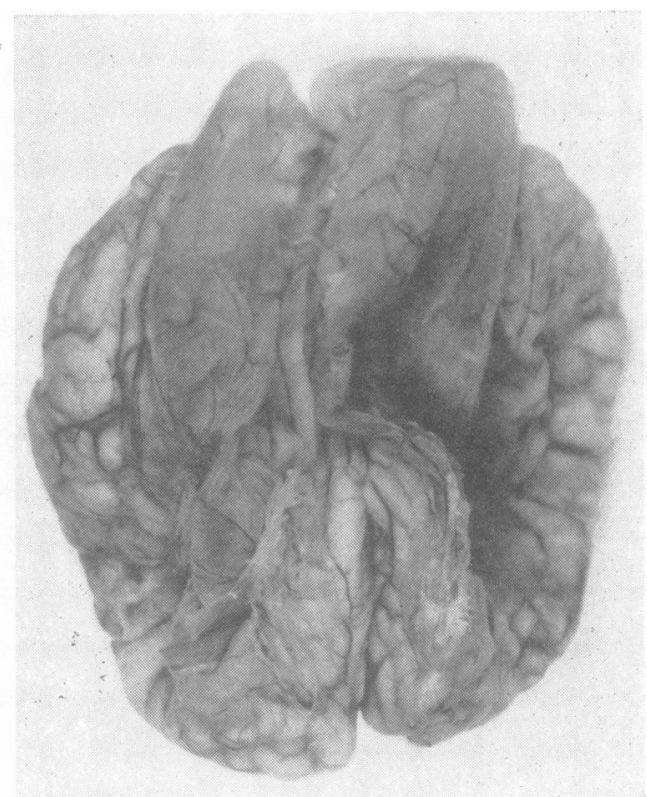

Fig. 2.-Brain, convexity: the frontal and greater part of the parietal lobes are replaced by a thin-walled bladder. 
FIg 3.-Coronal section of brain: preservation of temporal lobes and dilatation of lateral ventricles. Heidenhain $\times 1.25$.

gyri are preserved. The left middle temporal and both lower temporal, fusiform, and hippocampal gyri, as well as both temporal poles, are preserved.

Coronal sections show gross dilatation of the lateral ventricles which occupy the entire membranous bladder (Fig. 3). The posterior and inferior horns, which lie in

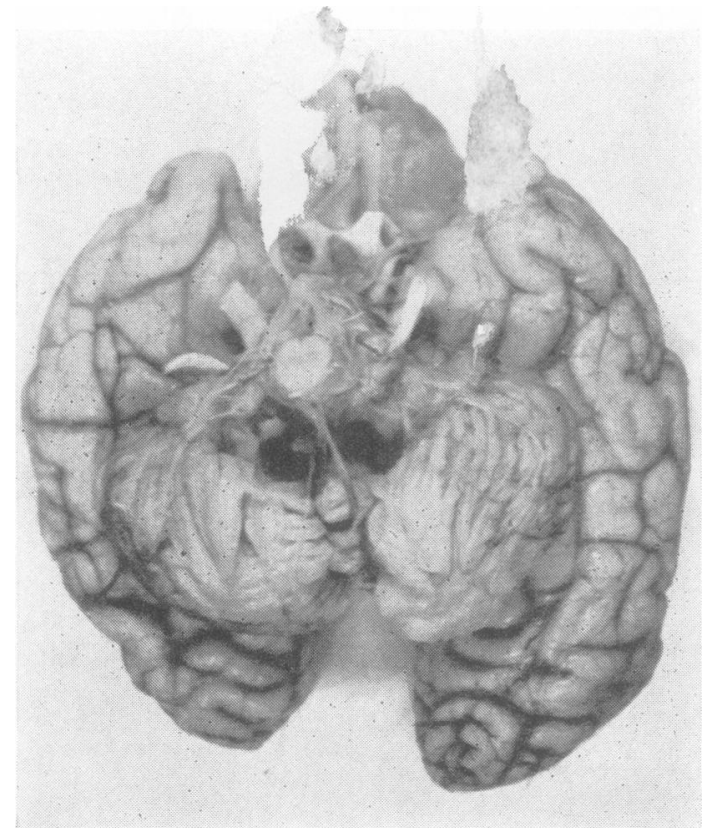

Fig. 4.-Brain, base: small cerebellum and widely gaping fourth ventricle. relatively well preserved parts of the brain, are also $\overline{0}$ grossly dilated and the subcortical white matter in thes 응 parts is considerably thinned. The ventricular surface rough and distinctly granular in places. The thir\& ventricle is also considerably dilated. if

The basal ganglia are small and atr $\lrcorner$ phic and detaiks of structure are not discernible. The corpus callosum is not identifiable except for a thin strand at the splenium

The brain-stem shows small and atrophic cerebra peduncles, pes pontis, and medullary pyramids. Other- of wise its structure appears normal. The aqueduct and the fourth ventricle are considerably dilated.

The cerebellum is small but shows no obvious structural abnormality. The posterior medullary velum is absent and the grossly dilated fourth ventricle communicates directly with the cisterna magna (Fig. 4).

The cranial nerves appear normal. The spinal cord is somewhat thinner than normal, but shows no obvious lesion.

\section{Histology}

The cerebral hemispheres were divided into several coronal blocks, embedded in celloidin and stained with carbol-azure, Heidenhain's method for myelin, and Mallory's phosphotungstic-acid-haematoxylin. The blocks containing the basal ganglia were cut serially and every fortieth section examined. Representative sections of the brain-stem, cerebellum, and spinal cord were embedded in celloidin and stained similarly. In addition frozen sections of the cerebellum were stained with Holzer's method for glia and with Cajal's silver impregnation. The arteries o of the circle of Willis were embedded in paraffin and $N$ cut serially, every tenth section stained with haema- N 


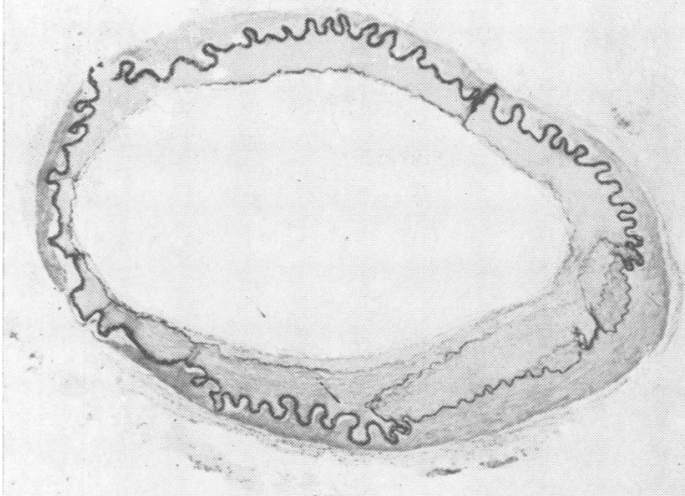

FIG. 5.-Right internal carotid artery: intimal fibrosis and splitting of internal elastica. Verhoeff-van Gieson $\times 35$.

toxylin and eosin and selected adjoining sections with Masson's green trichrome and with VerhoeffVan Gieson's stain.

Blood Vessels. - In its intracranial course the right internal carotid artery shows a considerable degree of intimal thickening, crescentic in shape, and consisting of circularly arranged collagen fibres with scanty ce " tig. 5). Underneath the point of maximal thic nness of the intima the internal elastic lamina is disorganized and split into several thin, ill-defined lamellae. Elsewhere the internal elastica is well preserved and an additional thin sheet of eiastica can be seen beneath the endothe-

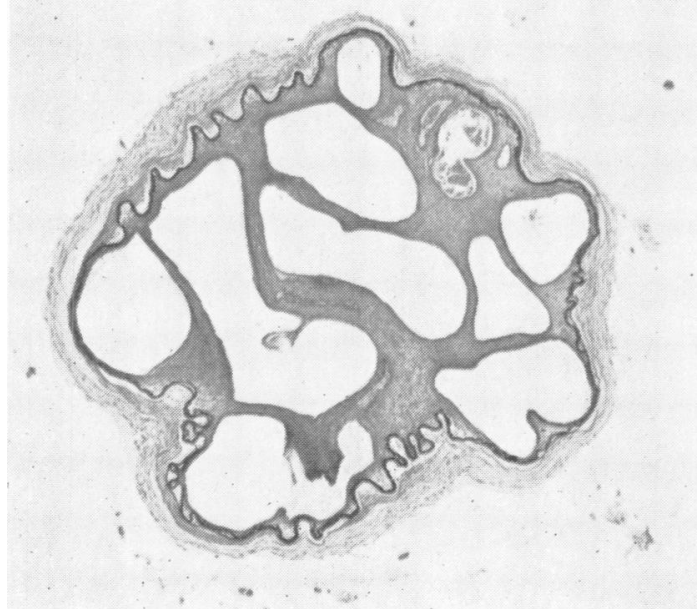

FIG. 6.-Right middle cerebral artery: Multilocular recanalization of occluded lumen. Verhoeff-van Gieson $\times 35$. lium. The media is thin and contains an excess of collagen fibres. The adventitia shows slight fibrous thickening.

The left internal carotid is similarly affected, but the intimal thickening is less pronounced and the internal elastica, though split in places, is less disorganized.

Of the arteries of the circle of Willis, the two anterior an. he two middle cerebral arteries show the most sev lesions. Near their points of origin their lumina are filled with a meshwork of fibrous

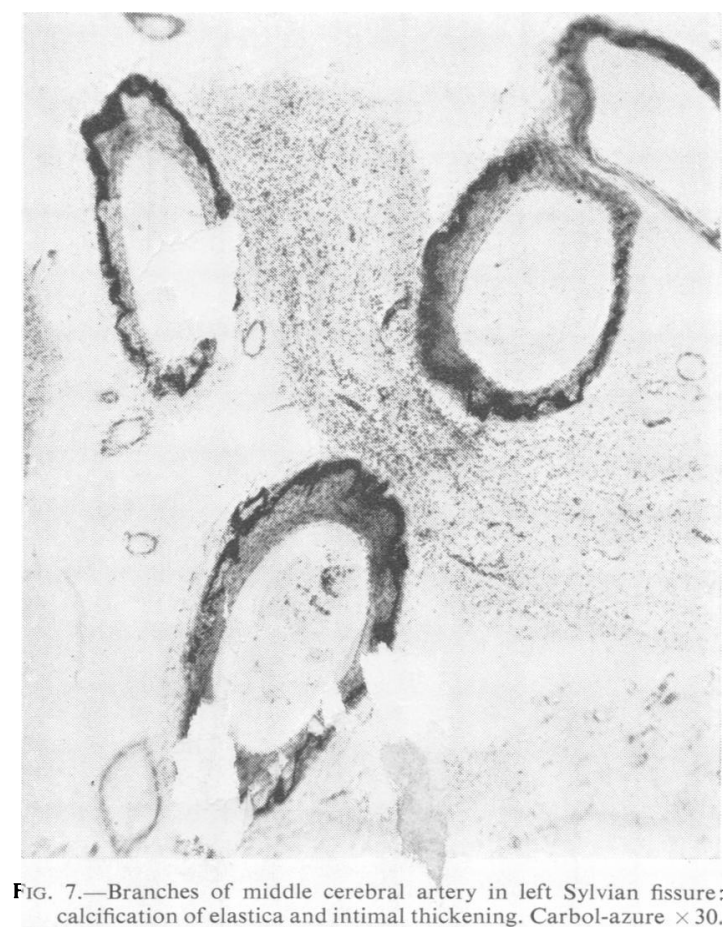

trabeculae, traversed by numerous channels lined with endothelium and surrounded by a thin layer of smooth muscle fibres (Fig. 6). The internal elastica is relatively well preserved, but shows thinning, fragmentation, and splitting in places. The media is completely destroyed and the fibrous adventitia begins immediately underneath the elastica. In the more peripheral course of the arteries the channels unite into a single lumen surrounded by a ring of intimal fibrosis, the media reappears and the lesions resemble those described in the internal carotids.

The posterior communicating arteries show only localized patches of intimal thickening with formation of an additional subendothelial elastic layer. The right posterior cerebral artery shows mild 
intimal fibrosis only. The left posterior cerebral artery is normal.

The medium-sized subpial, and to a lesser extent, intracerebral branches of the anterior and middle cerebral arteries show extensive calcification of the elastica in addition to severe intimal and adventitial fibrosis (Fig. 7). The smaller vessels show considerable, mainly adventitial, fibrosis. In the areas of complete destruction some of the small vessels show considerable narrowing of their lumina down to complete obliteration.

The medium and small arteries of the posterior cerebral territory, the brain-stem, and the cerebellum show no lesions. The veins appear normal.

Meninges.-The leptomeninges show a variable degree of thickening with proliferation both of the arachnoid endothelium and of collagen fibres. Here and there there are traces of lymphocytic infiltration. These lesions are very irregularly distributed and show no sites of predilection.

Cerebral Cortex.-The frontal cortex is completely destroyed and represented by a thin membrane consisting of four layers (Fig. 8). The outer layer is formed by thickened leptomeninges. The second layer consists of the remnants of the outer layers of the cortex which is devoid of nerve cells and shows considerable astrocytic proliferation and a moderate degree of fibrous gliosis, particularly in the marginal zone. This layer varies considerably in thickness. In some areas it is very thin, and even broken up in places, in others islands of somewhat thicker tissue can be seen, often with patches of spongy rarefaction in the centre. The third layer consists of a loose network of collagen fibres with numerous small, partly obliterated blood vessels. In the meshes of the network clusters of fat-laden microglial cells can be seen. The fourth layer, which represents the ventricular wall, consists of a thin, almost linear collagenous membrane (" pia accessoria ") denuded of ependyma. The central, insular and large part of the parietal cortex is similarly affected. Towards the edge of the lesion the subpial glial layer gradually increases in width and small groups of nerve cells appear in the scar tissue. Some of these cells are sclerotic, some are calcified (ferruginated). The pia accessoria also expands into a thicker glial layer, resembling the subpial one, and traversed by bundles of collagen fibres. Small islands of ependymal cells reappear on the ventricular surface. This transitional zone of ulegyria leads to the well-preserved occipital cortex which shows a normal cytoarchitectural pattern, and only a mild degree of marginal gliosis. Some remnants of cortical tissue also reappear in the posterior part of the cingulate gyrus. In the temporal lobes the lesions

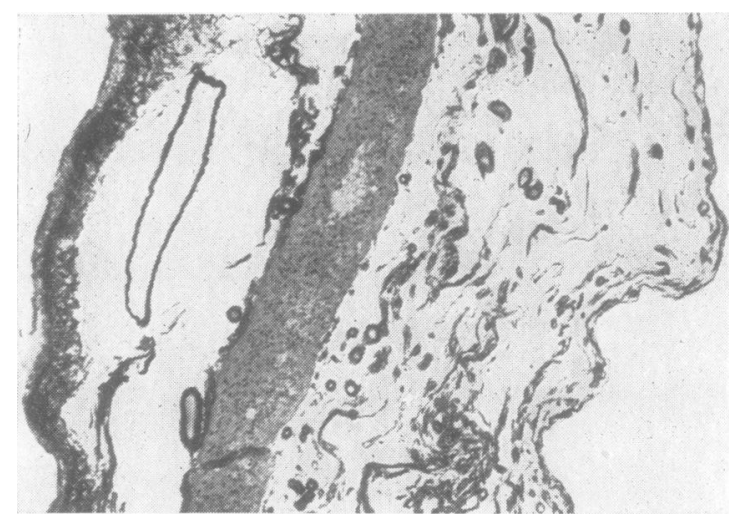

FIG. 8.-Membranous wall of bladder: four-layer structure consisting of leptomeninges, cortical remnant, fibro-vascular network, and "pia accessoria". Van Gieson $\times 40$.

are slightly asymmetrical, and are confined to the superior temporal gyrus on the left side, and to the superior and middle gyri on the right.

The hippocampal allocortex also shows more severe damage on the right side, where there is an area of cell loss in Sommer's sector of Ammon's horn with replacement gliosis and also some cello loss in the pyramidal layer of the uncus, as well as尺 considerable destruction of the amygdaloid nucleus. $\mathbb{\Omega}$ On the left side there is only slight cell loss in Sommer's sector with fibrous gliosis. The left amygdaloid is preserved. The fornix can be traced on both sides. It is thin, but contains myelinated fibres, more numerous on the left than on the right. The corpus callosum and anterior commissure are not identifiable.

The subcortical white matter is completely destroyed in the areas corresponding to the membranous cortex. Where the cortex is preserved in the occipital and temporal lobes the white matter shows considerable thinning but is well myelinated. The white matter in the roof of the right inferior horn is destroyed and replaced by a glial scar. On the left side it is preserved and both the fibres of the optic radiation and the bundle of Türck are identifiable.

Basal Ganglia.-The striatum is destroyed except for a small postero-ventral part of the putamen and the tail of the caudate which can be identified in its course along the inferior horn of the lateral ventricle on both sides. Only the posterior half of the right pallidum is represented, and this shows considerable cell loss, severe loss of myelin, and heavy gliosis. The nuclei basales are intact.

The thalamus is shrunken and distorted and identification of the surviving cell groups is difficult. The main cell masses of the anterior, medial, and 
lateral nucleus have almost completely disappeared and are replaced by dense glial scars. Of the surviving nuclei the paraventricular and habenular can be identified. Of the centromedian group the medial part, which lies in close apposition to the fasciculus habenulo-interpeduncularis (the nucleus parafascicularis), is well preserved while the centrum medianum proper is largely destroyed and represented only by a few scattered cells. The nucleus lateralis dorsalis and possibly also the adjoining part of the medial nucleus of the pulvinar is preserved on both sides. The nucleus pulvinaris inferior (the intergeniculate nucleus) is preserved on the left side, partly damaged on the right. The thalamus shows severe loss of myelinated fibres and in its posterior part the well preserved habenulo-interpeduncular tract stands out conspicuously. Myelinated fibres are also present in the posterior commissure and in the stria medullaris.

The left lateral geniculate body appears intact; the right is not identifiable. The optic chiasm and both optic tracts are well myelinated, but the right is slightly smaller than the left. The medial geniculate bodies are severely atrophied.

In the subthalamic region both bodies of Luys are present, but show some damage. The fields of Forel and the zona incerta are prominent.

The hypothalamus is remarkably well preserved from the neighbourhood of the optic chiasm backwards. The supra-optic and paraventricular nuclei are conspicuous on both sides. The left mammillary body appears normal; the right has been damaged by artefact. The fibres of the column of the fornix can be seen entering the mammillary body, but the tract of Vicq d'Azyr is not traceable. The nuclei of the tuber and infundibulum appear normal. The pituitary stalk shows no lesion.

The pre-optic fields and the septal nuclei are preserved. The nucleus accumbens is destroyed on both sides.

Of the internal capsules only the postero-inferior part of the left capsule containing the optic radiation and the temporo-pontine fibres is preserved.

Brain-stem.-The nuclei of the cranial nerves and the tegmental nuclei are intact. The red nucleus and substantia nigra appear normal. There is no apparent cell loss in the quadrigeminal bodies. The cerebral peduncles are shrunken and demyelinated except for the intact left temporo-pontine and partly damaged right temporo-pontine tracts. The pontine nuclei are mildly atrophied, the cell loss being more conspicuous on the right. The pyramidal tracts are completely demyelinated throughout their cours through the brain-stem. The medial lemniscus, the medial longitudinal bundle, and the brachia con- junctiva are well myelinated. In the lower medulla the nuclei graciles show cell loss, neuronophagia, demyelination, and gliosis. The cuneate nuclei and their fibres are normal.

Cerebellum.-The cortex shows a diffuse granular layer atrophy of neocerebellar distribution (Fig. 9).

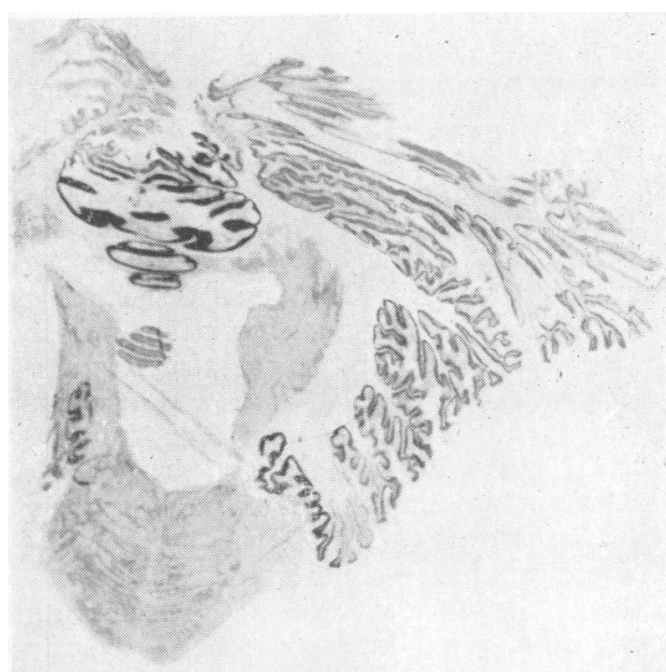

FIG. 9.-Cerebellum: predominantly neocerebellar distribution of atrophy. Carbol-azure $\times 1.5$.

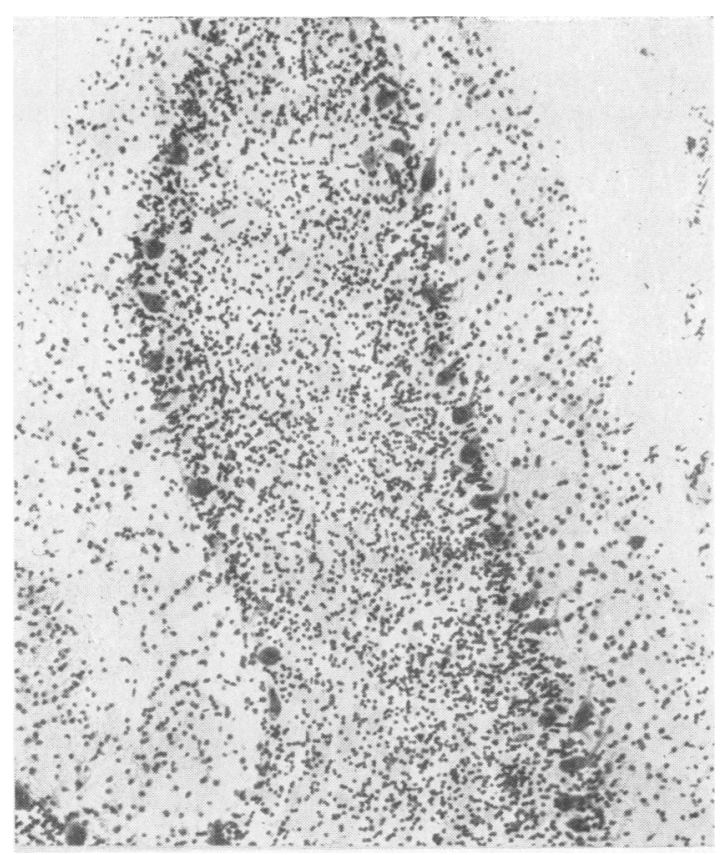

Fig. 10.-Cerebellum: rarefastion of granular layer with preservation. of Purkinje cells. C.rbol-azurs $\times 100$. 
The granular layer is thin and rarefied, while the Purkinje, Golgi, and stellate cells are intact (Fig. 10). The various fibre systems are also well preserved. In some places, however, complete atrophy of the cerebellar cortex can be seen. In a few folia this is confined to the depths of the sulci, in a few others the tips of the folia are the more affected. At the edges of these areas of total destruction some disorganization of cortical architecture can be observed, accompanied by displacement of Purkinje cells into the molecular layer. Degenerative forms of Purkinje cells with axonal torpedoes and cactus-like swellings of the dendrites can occasionally be seen. These areas of destruction and disorganization show a heavy fibrous gliosis with proliferation both of radial and tangential glial fibres. On the other hand, in areas which only show granular rarefaction, gliosis is conspicuously absent. The central cores of the folia show demyelination and gliosis only in the areas of complete cortical atrophy. Where the lesion affects the tips of the folia this may be associated with status spongiosus or even frank cavitation of the white core.

The cortex of the vermis is normal.

There is a patch of cell loss in the posterior part of the dentate nucleus accompanied by partial demyelination in the amiculum. Elsewhere the nuclei are well myelinated.

Spinal Cords.-At the lower cervical level there is a small wedge-shaped lesion in the posterior columns, completely replacing the tracts of Goll and encroaching upon the tracts of Burdach (Fig. 11). The highly cellular centre of the lesion consists of numerous swollen, proliferated, occasionally multinuclear astrocytes, numerous oligodendro- and microglial cells and a few perivascular histiocytes and fibroblasts. Fat-laden microglial cells are fairly numerous, particularly at the edge of the lesion. There is considerable proliferation of small blood vessels with a thick fibrous adventitia. This central

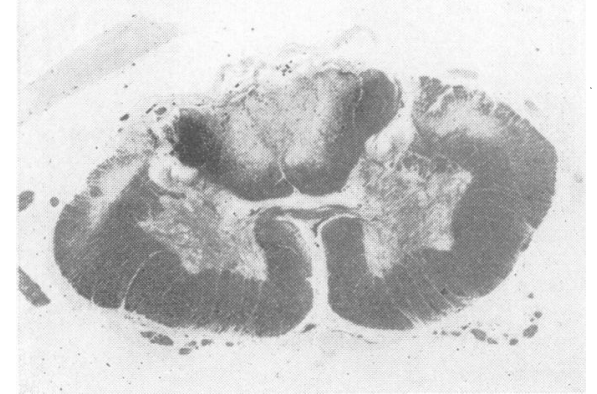

Fig. 11.-Lower cervical cord: areas of demyelination in posterior and lateral columns. Heidenhain $\times 5$. lesion is surrounded by a zone of rarefied nervous tissue with a few myelinated nerve fibres, numerous astrocytic nuclei, but only very scanty glial fibres. This merges gradually into the healthy tissue of the lateral parts of the tracts of Burdach. The lateral columns show demyelination in the areas corresponding to the crossed pyramidal tracts. In addition the posterior halves of the lateral columns are the site of an active glio-mesodermal reaction similar to, but less intense than that found in the posterior columns. The central grey matter is normal.

At lower levels (mid-thoracic and mid-lumbar) demyelination of the pyramidal tracts is the only abnormality seen.

Ventricular System.-The entire ventricular system of the brain is grossly dilated. In the membranous parts of the fore-brain the ependyma is absent and the ventricular surface is lined by a thin fibrous membrane. In the areas where the wall of the lateral ventricle is preserved, as well as in the third and fourth ventricles and the aqueduct, the ependyma is broken up by numerous nodules of proliferated fibrous glia. In many places groups of ependymal cells are buried under the confluent glial nodules, where they form rosettes and tubular structures. In some places, particularly in the posterior horn of the lateral ventricles, the lining is formed by a layer of thick collagen fibres, interspersed here and there with glial fibres. The choroid plexuses are normal.

\section{Discussion}

In this case the intensity of the infection was originally damped down by the systemic chemotherapy, with the result that the child was able to survive for nearly five weeks. That, at the end of this time, the infection was nevertheless still active was shown unequivocally by the isolation of typable pneumococci from the C.S.F. During these weeks the clinical picture had changed from that typical of acute pneumococcal meningitis to that of posterior basic meningitis of infants, a condition classically associated with low-grade meningococcal infections. Moreover, the obstructive hydrocephalus had reached a pitch at which the high intracranial pressure constituted an immediate threat to life.

The infection was permanently cured by appropriate chemotherapy and, as a result of this and of surgical intervention (Cairns, 1949), the obstructive hydrocephalus was relieved. Nevertheless, the child remained a hopeless idiot. It was considered at that time that the likeliest explanation for this gross residual deficit was widespread cerebral infarction secondary to purulent arteritis (Cairns, 1947, 1949).

Since that time an identical picture has been seen several times in cases of tuberculous meningitis in 
which treatment was not begun until late in the disease (Smith and Vollum, 1950; Smith, Vollum, Taylor, and Taylor, 1956), and where destruction of brain tissue consequent on an advanced arteritis was the essential lesion.

The post-mortem findings confirmed this clinical impression. Most of the lesions were either direct or remote consequences of arterial occlusion. A typical post-infective hydrocephalus was also present. In addition an apparently unrelated lesion in the cervical cord and an unusual type of cerebellar atrophy were found.

Vascular lesions in the acute and subacute stages of pneumococcal meningitis have been described by Cairns and Russell (1946). The findings in the present case represent the final residual changes of the processes described by these authors. There is, however, one essential difference in the distribution of the lesions: while in Cairns and Russell's cases only the medium and small subpial and intracerebral vessels were affected, in our case the most severe damage was found in the main arterial trunks as they traversed the arachnoid cisterns at the base of the brain.

The anterior and middle cerebral arteries on both sides showed evidence of occlusion by an obliterative process, either proliferative or thrombotic which had undergone recanalization by multiple intercommunicating channels. Not only the lumen but also the entire arterial wall had become considerably disorganized. The media showed loss of muscle fibres, complete in places, and replacement by fibrous tissue continuous with that of the thickened adventitia. The internal elastica had proved to be most resistant to the destructive process, but it also showed signs of damage in the form of thinning, fragmentation, and splitting into thin, irregularly distributed lamellae. New layers of elastica could also be seen in the intimal fibrous tissue. All these lesions appeared to be the end-result of an obliterative panarteritis and resembled in all essential features the arteritis described by Heubner (1874) in cerebral syphilis.

The calcification of the internal elastica was probably the end-result of fibrinoid necrosis described by Cairns and Russell in the subacute stage. It might be, as these authors suggest, due either directly to infection, or to tissue sensitization caused by repeated reinfection of the cerebrospinal pathway. Alternatively, Meyer (1951), who observed similar lesions in cases of birth injury without evidence of infection, ascribes them to ischaemic damage of the arterial wall. The fact that this change in our case was present only in arteries distal to the occlusion lends weight to this point of view. We have also found identical lesions in a case of arterial occlu- sion in early childhood (Norman and Urich, 1957).

The occlusion of a number of small arterial branches, particularly in infarcted areas, might be the end-result of an inflammatory process, or more likely, the physiological obliteration of nonfunctioning blood vessels.

In striking contrast to the arteries the veins appeared to have escaped damage. This is in agreement with the observations of Cairns and Russell, who found lesions confined almost entirely to arteries in the subacute stage, although in the acute phase both types of vessel seemed to be equally affected.

The occlusion of the two main branches of the internal carotids on both sides had caused infarction of the entire territories of these arteries. The infarcted parts of the pallium had been reduced to a thin, semitransparent membrane giving the brain the appearance encountered in some cases of hydranencephaly. The histological appearance of the membrane, however, differed from that seen in true hydranencephaly in which the fibrovascular network is absent implying destruction and reabsorption in an earlier stage of development (Lange-Cosack, 1944).

The distribution of lesions in the basal ganglia corresponded closely with the extent of the cortical and subcortical lesions. The striatum was completely destroyed with the exception of the part of the putamen and the tail of the caudate which receive their blood supply from the anterior choroidal artery. The internal and posterior parts of the pallidum supplied by the same artery had also survived though not unscathed. The heavy gliosis of this nucleus is not surprising in view of the fact that it forms part of the wall of an old infarct cavity. Lesions in the territory of the anterior choroidal artery were limited to vulnerable areas like Sommer's sector of Ammon's horn, the pyramidal layer of the uncus, and the amygdaloid nucleus, all lesions of a non-specific character, commonly observed in epilepsy. It is difficult to be certain whether this preservation of the anterior choroidal territory was due to the fact that the artery had escaped occlusion or to its abundant anastomoses with branches of the posterior cerebral artery.

The extensive thalamic lesions did not appear to be the result of direct ischaemic damage, the blood supply of the thalamus being derived mainly from the posterior cerebral and posterior communicating arteries with some contribution from the anterior choroidal artery, while the middle cerebral component was inconstant and insignificant (Foix and Hillemand, 1925). The damage is due to retrograde atrophy of the relay nuclei caused by destruction of the cerebral cortex and the striatum. Most 
thalamic nuclei are known to have end-brain projections and total ablation of the end-brain results in atrophy of the thalamus with the exception of the habenular, paraventricular, and parataenial nuclei (Rose and Woolsey, 1949). The first two of these were preserved in the present case, but the parataenial, which is very inconspicuous in man (Dekaban, 1953), could not be identified. In a case of hemiatrophy of the cerebral cortex, Le Gros Clark and Russell (1940) found the centromedian nucleus preserved in an otherwise almost completely atrophied thalamus. The striatal projection of this nucleus is now well established (McLardy, 1948). The medial part of this nuclear group, known as the parafascicular nucleus, was thought by Simma (1951) to project on to the nucleus accumbens ("fundus striati "). This was denied by Powell and Cowan (1956), who showed that in the monkey the parafascicular nucleus projected on to a part of the putamen while the parataenial projected on to the accumbens. The findings in the present case support the views of these authors and suggest that the connexions of these nuclei may be similar in man. Of the cortical relay nuclei only two remained: the nucleus pulvinaris inferior and the nucleus lateralis dorsalis. The inferior nucleus of the pulvinar, also known as the intergeniculate nucleus, projects on to the para- and peristriate areas of the occipital cortex while the main mass of the pulvinar sends fibres mainly to the lips of the Sylvian fissure (Le Gros Clark and Northfield, 1937). The projection of the nucleus lateralis dorsalis is still a subject of controversy. Walker (1938) suggests tentatively that this nucleus may project on to the upper lip of the Sylvian fissure, but admits that the experimental evidence is not entirely convincing. The findings in the present case suggest that this view is incorrect, at least for man. Kuhlenbeck (1954) maintains that both the dorsal and the posterior lateral nuclei project on to the posterior parietal cortex in man. This cannot be ruled out in view of the survival of the superior parietal lobule, but does not agree with the results of the experimental work either of Le Gros Clark and Boggon (1935) or of Walker (1938).

Retrograde atrophy must also be held responsible for the destruction of the medial geniculate and of the right lateral geniculate bodies. The destruction of the latter was due to interruption of the fibres of the optic radiation in the roof of the inferior horn of the lateral ventricle, and not to damage of the visual cortex, which was intact. On the left side the optic radiation was preserved and so was the lateral geniculate body. The hypothalamus, which derives its blood supply from the posterior communicating arteries, was intact.

Contrary to the findings of Cairns and Russell, no vascular lesions could be seen in the brain-stem. The long descending tracts of cortical origin were degenerated except for the left temporopontine tract. The lesion affecting the posterior and, to a lesser extent, the lateral columns of the cervical cord was not coeval with the other lesions. Its appearances suggested a recent necrosis with an active and intense glio-mesodermal reaction. The nature of this lesion is obscure, but it resembles sufficiently those described in cervical spondylosis (Bedford, Bosanquet, and Russell, 1952; Greenfield, 1953; Mair and Druckman, 1953) to suggest that it also was traumatic in origin. It is conceivable that subluxation of the cervical spine might occur in a totally paralysed idiot with extreme opisthotonus even in the course of ordinary nursing procedures. We have observed a similar lesion in another case of severe cerebral palsy.

The hydrocephalus showed all the characteristic features of post-inflammatory hydrocephalus as described by Russell (1949). It involved all four ventricles and the aqueduct. The exact site of obstruction could not be established at necropsy as a large part of the roof of the fourth ventricle had been removed surgically to relieve occlusion of the foramina. In spite of the relief afforded the damage appears to have remained irreversible. Severe granular ependymitis and collagen replacement of the ependyma of the posterior horn, common accompaniments of post-meningitic hydrocephalus, were also observed in this case.

The cerebellar lesions fall into two groups. selective atrophy of the granular layer and lobular atrophy involving both the granule and the Purkinje cells. In the present case it is difficult to account for the selective loss of granule cells except upon the basis of a toxic effect occurring at the time of the acute infection. Williams (1934) first drew attention to "conglutination" of the granular layer in individuals dying in the acute stage of septic meningitis and considered the lesion to be analogous to that produced by experimental poisoning in animals. It has to be admitted, however, that atrophy of the granule cells has not been hitherto described as a late sequel of acute meningitis and this suggests that conglutination, when not an artefact, may be a reversible lesion and that in this instance it has indeed progressed to a stage of permanent neuronal damage. Alternative explanations appear to be less plausible. For example, it would perhaps be tempting to ascribe the predominantly neocerebellar distribution of the lesion to secondary degeneration following the loss of the corticopontine tracts. In the reported cases of crossed cerebellar atrophy, however, the Purkinje cell layer has always been conspicuously involved (Verhaart 
and van Wieringen-Rauws, 1950). A mainly neocerebellar distribution of atrophy of the granular layer has been described in other conditions, evidently unrelated to the present case. It may be found in a congenital type of cerebellar affection, as described by Ule (1952). Here the marked heterotopia of Purkinje cells and their frequent maldevelopment are distinctive features. The destruction of the granular layer due to poisoning by organic mercury compounds (Hunter and Russell, 1954) is of special interest because the effect of an exogenous toxin is so clearly demonstrated. In their case the sparing of the palaeocerebellum was considered remarkable.

The much less conspicuous and patchily distributed atrophy of individual lobules and folia in the cerebellum of our case may also be related, at least in part, to the original infection. Noetzel (1944) described lobular sclerosis in children who survived an attack of acute suppurative meningitis. In his cases the tips of the folia were more affected and he suggested that the lesions were due to a spread of the infection from the meninges. With regard to the lobular atrophy in the deeper parts of the cerebellar cortex, the possibility that this was a post-epileptic change cannot be ruled out (Scholz, 1951), but it may well be that both pathogenetic mechanisms might have been operative.

\section{Summary}

A previously healthy girl aged 18 months developed pneumococcal meningitis. Treatment with sulphonamides failed to eradicate the infection but altered profoundly the course of the disease which came to resemble subacute posterior basic meningitis. Subsequent surgical procedures and treatment with penicillin brought the infection under control and relieved an obstructive internal hydrocephalus, but the child remained in a state of decerebrate rigidity in which she survived for 11 years.

At necropsy an old, partly recanalized, obliterative arteritis was found in both anterior and both middle cerebral arteries. Their territories of supply had undergone infarction and were replaced by a thin semitranslucent membrane. The thalamus showed extensive retrograde degeneration; of the nuclei with cortical projection, only the nucleus pulvinaris inferior and the nucleus lateralis dorsalis had survived. The cortical projection tract showed long-standing degeneration.

In addition, an unusual type of cerebellar degeneration was found, consisting predominantly of atrophy of the granular layer in the lateral lobes. A recent lesion in the lower cervical cord was also discovered; it was thought to be due to trauma, probably a subluxation of the cervical spine.

We wish to thank Dr. W. A. Heaton-Ward of Stoke Park Hospital, Bristol, for his kind permission to use his hospital records. This work was carried out with the assistance of the Nuffield Foundation.

\section{REFERENCES}

Bedford, P. D., Bosanquet, F. D., and Russell, W. R. (1952). Lancet, 2, 55 .

Cairns, H. (1947). Broin, 70, 251.

(1949). Brit. med. J., 1, 969.

- and Russell, D. S. (1946). J. Path. Bact. 58, 649.

Duthie, E. S., Lewin, W. S., and Smith, H. V. (1944). Lancet, 1, 655 .

Clark, W. E. Le Gros, and Boggon, R. H. (1935). Philos. Trans. B., 224, 313.

, and Northfield, D. W. C. (1937). Brain, 60, 126.

and Russell, D. S. (1940). Journal of Neurology, Neurosurgery and Psychiatry, 3, 123.

Dekaban, A. (1953). J. comp. Neurol., 99, 639.

Foix, C., and Hillemand, P. (1925). Rev, neurol (Pcris) 32 (2), 705.

Gee, S., and Barlow, T. (1878). St. Bart's Hosp. Rep., 14, 23.

Greenfield, J. G. (1953). Rev. med. Suisse rom., 73, 227.

Heubner, O. (1874). Die luetische Erkrankung der Hirnarterien, pp. 124-169. Vogel, Leipzig.

Hunter, D., and Russell, D. S. (1954). Journal of Neurology, Neurosurgery and Psychiatry, 17, 235.

Kuhlenbeck, H. (1954). "The Human Diencephalon". Suppl. to Confin. neurol. (Basel), XIV, p. 75.

Lange-Cosack, H. (1944). Arch. Psychiat. Nervenkr., 117, 1.

McLardy, T. (1948). Brain, 71, 290.

Mair, W. C. P., and Druckman, R. (1953). Ibid., 76, 70.

Meyer, J. E. (1951). Arch. Psychiat. Nervenkr., 186, 437.

Noetzel, H. (1944). Ibid., 117, 275.

Norman, R. M., and Urich, H. (1957). J. Path. Bact., 73, 580.

Powell, T. P. S., and Cowan, W. M. (1956). Brain, 79, 364 .

Rose, J. E., and Woolsey, C. N. (1949). Electroenceph. clin. Neurophysiol., 1, 391.

Russell, D. S.'(1949). Rep. Ser. med. Res. Coun. (Lond.), No. 265.

Scholz, W. (1951). Die Krampfschädigungen des Gehirns, pp. 29-34. Springer, Berlin.

Simma, K. (1951). Mschr. Psychiat. Neurol., 122, 32

Smith, H. V. (1951). Practitioner. 166, 334.

Smith, Huthie, E. S., and Cairns, H. (1946). Lancet, 1, 185. Duthie, E. S., and Cairns, H. (1946).

Vollum, R. L., Taylor, L. M., and Taylor, K. B. (1956). Tubercle (Lond.), 37, 301

Ule, G. (1952). Disch. Z. Nervenheilk., 168, 195.

Verhaart, W. J. C., and Wieringen-Rauws, G. A. van (1950). Folia

psychiat. (Amst.), 53, 481.
Walker, A. E. (1938). The Primate Thalamus, p. 185 . University of Chicago Press.

Williams, E. Y. (1934). Arch. Path. (Chicago), 17, 206. 